\title{
RNA interference targeting hypoxia-inducible factor $1 \alpha$ via a novel multifunctional surfactant attenuates glioma growth in an intracranial mouse model
}

\author{
David L. Gillespie, PhD, ${ }^{1}$ Maria T. Aguirre, MD, ${ }^{2}$ Sandhya Ravichandran, MD, ${ }^{2}$ \\ Lisa L. Leishman, MD, ${ }^{2}$ Claudia Berrondo, MD, ${ }^{3}$ Joseph T. Gamboa, BS, ${ }^{2}$ Libo Wang, BS, ${ }^{4}$ \\ Rose King, BS, ${ }^{5}$ Xuli Wang, PhD, ${ }^{6}$ Mingqian Tan, PhD, ${ }^{7}$ Anthony Malamas, PhD, ${ }^{7}$ \\ Zheng-Rong Lu, PhD, ${ }^{7}$ and Randy L. Jensen, MD, PhD ${ }^{1}$
}

\begin{abstract}
${ }^{1}$ Huntsman Cancer Institute and Department of Neurosurgery and ${ }^{2}$ School of Medicine, University of Utah, Salt Lake City, Utah; ${ }^{3}$ Dartmouth School of Medicine, Hanover, New Hampshire; "Department of Anesthesiology, University of Utah, Salt Lake City, Utah; ${ }^{5}$ Department of Biology, Truman State University, Kirksville, Missouri; ${ }^{6}$ Division of Radiobiology, University of Utah, Salt Lake City, Utah; and 'Department of Biomedical Engineering, Case Western Reserve University, Cleveland, Ohio
\end{abstract}

\begin{abstract}
OBJECT High-grade gliomas are the most common form of adult brain cancer, and patients have a dismal survival rate despite aggressive therapeutic measures. Intratumoral hypoxia is thought to be a main contributor to tumorigenesis and angiogenesis of these tumors. Because hypoxia-inducible factor $1 \alpha(\mathrm{HIF}-1 \alpha)$ is the major mediator of hypoxia-regulated cellular control, inhibition of this transcription factor may reduce glioblastoma growth.

METHODS Using an orthotopic mouse model with U87-LucNeo cells, the authors used RNA interference to knock down HIF-1 $\alpha$ in vivo. The small interfering RNA (siRNA) was packaged using a novel multifunctional surfactant, 1-(aminoethyl)iminobis[ $\mathrm{N}$-(oleicylcysteinylhistinyl-1-aminoethyl)propionamide] (EHCO), a nucleic acid carrier that facilitates cellular uptake and intracellular release of siRNA. Stereotactic injection was used to deliver siRNA locally through a guide-screw system, and delivery/uptake was verified by imaging of fluorescently labeled siRNA. Osmotic pumps were used for extended siRNA delivery to model a commonly used human intracranial drug-delivery technique, convectionenhanced delivery.
\end{abstract}

RESULTS Mice receiving daily siRNA injections targeting HIF-1 $\alpha$ had a $79 \%$ lower tumor volume after 50 days of treatment than the controls. Levels of the HIF-1 transcriptional targets vascular endothelial growth factor (VEGF), glucose transporter 1 (GLUT-1), C-MET, and carbonic anhydrase-IX (CA-IX) and markers for cell growth (MIB-1 and microvascular density) were also significantly lower. Altering the carrier EHCO by adding polyethylene glycol significantly increased the efficacy of drug delivery and subsequent survival.

CONCLUSIONS Treating glioblastoma with siRNA targeting HIF-1 $\alpha$ in vivo can significantly reduce tumor growth and increase survival in an intracranial mouse model, a finding that has direct clinical implications.

http://thejns.org/doi/abs/10.3171/2014.10.JNS132363

KEY WORDS RNA interference; HIF-1 $\alpha$; glioma; intracranial; tumor; mouse; siRNA; knockdown; oncology

$\mathrm{P}$ ATIENTS with the most malignant type of glioma, Grade IV glioblastoma, have a mean survival time of approximately 15 months and an estimated 5-year survival rate of less than 5\%.11 High-grade gliomas
(Grades III and IV) are the most common primary brain tumors in adults and the fourth leading cause of cancerrelated death..$^{40}$ Tumor hypoxia is thought to play an important role in glioblastoma tumor pathobiology by pro-

ABBREVIATIONS CA-IX = carbonic anhydrase-IX; CED = convection-enhanced delivery; DAB = 3,3'-diaminobenzidine tetrahydrochloride; DCA = dichloroacetate; EHCO = 1-(aminoethyl)iminobis[N-(oleicylcysteinylhistinyl-1-aminoethyl)propionamide]; GFP = green fluorescent protein; GLUT-1 = glucose transporter 1; HIF-1 $\alpha=$ hypoxia-inducible factor $1 \alpha$; IHC = immunohistochemistry; MVD = microvascular density; PEG = polyethylene glycol; PI = proliferation index; RNAi = RNA interference; siRNA = small interfering RNA; VEGF = vascular endothelial growth factor.

SUBMITTED October 28, 2013. ACCEPTED October 14, 2014.

INCLUDE WHEN CITING Published online November 28, 2014; DOI: 10.3171/2014.10.JNS132363.

DISCLOSURE The authors report no conflict of interest concerning the materials or methods used in this study or the findings specified in this paper. The following grant partially funded this study: Cancer Center Support Grant No. NIH P30 CA 42014. 
moting the development of highly vascularized tumors with chaotic and inefficient microcirculation. ${ }^{24,60}$ Indeed, hypoxic necrotic foci with pseudopalisading tumor cells are one of the features that define glioblastoma in the current World Health Organization classification scheme. ${ }^{28}$ Tumor hypoxia has been associated with resistance to radiotherapy and chemotherapy, as well as tumor invasion, in glioblastoma. ${ }^{7,47,50}$ Despite the use of aggressive chemotherapy, radiotherapy, and bevacizumab (an anti-vascular endothelial growth factor [VEGF] medication) therapy, clinical outcomes remain poor. ${ }^{44,58}$

Previous studies have shown that molecular markers of hypoxia, including the transcription factor hypoxiainducible factor $1 \alpha$ (HIF-1 $\alpha)$, and other hypoxia-regulated proteins, such as VEGF, carbonic anhydrase-IX (CA-IX), the receptor tyrosine kinase c-MET, and glucose transporter 1 (GLUT-1), $, 26,29$ are overexpressed in malignant brain tumors. ${ }^{54,66}$ HIF-1 $\alpha$ protein levels, tumor grade, and vascularity have been correlated in brain tumors, ${ }^{14,66}$ and studies have shown that hypoxia markers are predictive of outcomes for patients with a number of tumor types. ${ }^{17,22,37}$

The transcription factor HIF-1 is composed of 2 heterodimeric subunits, HIF- $1 \alpha$ and HIF-1 $\beta$. HIF-1 binds to DNA hypoxia-response elements (HREs) and induces the transcription of many well-characterized genes that help cells survive low-oxygen conditions. ${ }^{59}$ In addition to VEGF, these genes include erythropoietin, transferrin and its receptor, GLUT-1, and almost every gene in the glycolytic pathway. ${ }^{15}$ The activation of HIF-1 $\alpha$ promotes cell survival via an adaptive modification of cellular metabolism that increases these glycolytic enzymes and, hence, the glycolysis rate. This adaptation of cancer cells through increased glycolysis was first proposed by Otto Warburg ${ }^{62}$ as a necessary step toward an aggressive phenotype. ${ }^{53}$ Recent studies of HIF-1 have indicated a possible link with the Warburg effect in various cell types ${ }^{46,65}$ and prompted the hypothesis that aerobic glycolysis can be controlled by dysregulation of HIF-1 $\alpha .{ }^{36}$

At the mRNA level, HIF-l $\alpha$ and HIF-1 $\beta$ are both constitutively expressed and do not seem to be modified significantly by hypoxia. ${ }^{52}$ Although the HIF-1 $\beta$ protein is present in normoxic cells $\left(20 \% \mathrm{O}_{2}\right)$, HIF- $1 \alpha$ is almost undetectable under these conditions. In normoxia, HIF-1 $\alpha$ is degraded rapidly by proteasomal degradation through a mechanism that involves targeting by the von HippelLindau (VHL) protein. In low-oxygen-tension conditions $\left(1 \%-2 \% \mathrm{O}_{2}\right)$, this degradation is inhibited, leading to an increased level of HIF-1 $\alpha$, which translocates to the nucleus to activate gene transcription. ${ }^{45}$

The central roles of HIF-1 in the hypoxic response, angiogenesis, and glycolysis have made it a popular subject for cancer research in many different tumor types. Several preclinical research projects developing HIF-1 inhibitors are underway (reviewed by Onnis et $\mathrm{al}^{42}$ ), and a Phase I clinical trial investigating the efficacy of dichloroacetate (DCA) as an HIF-1 inhibitor in patients with glioblastoma and other recurrent brain tumors (ClinicalTrials.gov identifier, NCT01111097) is ongoing.$^{49}$ Many of these preclinical research studies involve viral or plasmid-mediated RNA interference (RNAi) that may pose serious risk or be ineffectual if used in humans. Although DCA is able to cross the blood-brain barrier, it is not a specific HIF-1 inhibitor but instead seems to function indirectly through pyruvate dehydrogenase kinase II deactivation..$^{55}$ Because glioblastomas can eventually escape from specific therapies, it is vital to pursue multiple treatments that target different points in essential pathways and can be combined (e.g., DCA and HIF-1-specific RNAi).

The development and application of clinical RNAi have been impeded by the lack of efficient and specific therapeutic small interfering RNA (siRNA) delivery systems for systemic administration. ${ }^{4,10,27}$ The plasma half-life of naked siRNA is only a few minutes after intravenous administration. ${ }^{1}$ To achieve clinically effective RNAi activity, suitable delivery reagents are required to protect siRNA from degradation and deliver siRNA to targeted cells. The current delivery systems are adopted mainly from nonviral genedelivery systems (e.g., cationic lipids, polymers, peptides, and aptamers ${ }^{27}$ ), but these systems are either toxic or inefficient for systemic delivery of therapeutic siRNA. Efficient systemic and targeted siRNA-delivery systems are needed for broad clinical applications of RNAi. Recently, a novel class of multifunctional surfactants with $\mathrm{pH}$-sensitive amphiphilicity for efficient siRNA delivery was designed and developed. ${ }^{60,61}$ One of these surfactants, 1-(aminoethyl) iminobis [ $N$-(oleicylcysteinylhistinyl-1-aminoethyl)propionamide] (EHCO), was used in the present study. A distinct advantage of this carrier is its $\mathrm{pH}$-sensitive amphiphilicity and amphiphilic cell-membrane disruption at an endosomal-lysosomal $\mathrm{pH}$, which can facilitate the specific endosomal-lysosomal release of an siRNA into the cytoplasm..$^{60}$ This surfactant can also be modified to improve stability for extended delivery times by adding polyethylene glycol (PEG). This addition facilitates extended siRNA delivery through an osmotic pump modality that mimics convection-enhanced delivery (CED), in that it provides positive pressure and continuous flow of the pump contents directly into the tumor through a cannula.

In this study, we used EHCO for the delivery of antiHIF-1 $\alpha$ siRNA by stereotactic injection or osmotic pumps to treat established intracranial tumors in vivo. Using bioluminescence imaging methods to monitor tumor growth and immunohistochemistry (IHC) to monitor tumor markers for angiogenesis, glycolysis, cell differentiation, and proliferation, we evaluated whether the intratumoral delivery of siRNA protected with EHCO to knock down HIF-1 $\alpha$ results in significant suppression of tumor growth in a mouse model of glioblastoma.

\section{Methods \\ Cell Culture and Transfection}

U87-LucNeo cells were maintained in DMEM with the standard addition of glucose, glutamine, sodium pyruvate, and $10 \%$ fetal bovine serum with $300 \mu \mathrm{g} / \mathrm{ml} \mathrm{G} 418$ for selection. These cells express luciferase constitutively and have been described previously. ${ }^{18}$ EHCO-siRNA and PEGylated EHCO-siRNA nanoparticles were prepared according to previously reported methods. ${ }^{60,61}$ U87 cells were also cultured for live-cell confocal imaging to analyze the intracellular presence and release of siRNA 48 hours after transfection with EHCO-siRNA. To accom- 
plish this process, approximately 50,000 U87 cells were initially seeded onto glass-bottom dishes (MatTek). Once $25 \%$ confluency was reached, the cells were transfected with 100 nM Alexa Fluor 488-labeled nonspecific siRNA in fresh serum-free medium. Nanoparticles were formulated at 12 protonable amines per phosphate group $(\mathrm{N} / \mathrm{P})$ by mixing together the proper molar amounts of EHCO and siRNA for a 30-minute period. The EHCO carrier was stored in ethanol at a $2.5 \mathrm{mM}$ concentration, whereas the siRNA was stored at a concentration of $18.8 \mu \mathrm{M}$ in nuclease-free water. Setting the ethanol/water volume ratio to 1:20 allowed for the formulation of stable complexes.

After a 4-hour incubation period, the EHCO-siRNA nanoparticles were aspirated and replaced with normal complete growth medium. The cells were then allowed to grow for an additional 48 hours after transfection. At this point, $75 \mathrm{nM}$ LysoTracker Red DND-99 (Invitrogen) was added to the culture dish in normal growth medium for 30 minutes. The cells were then rinsed twice and imaged using an Olympus FV1000 confocal microscope at $\times 1000$ magnification.

\section{Intracranial Brain Tumor Model}

Guide-Screw Implant

All animal experiments were performed under review of the University of Utah Animal Care and Use Committee. Four-week-old CD1-nu/nu mice were used following the protocol of Lal et al. ${ }^{31}$ with some modifications made by Brockmann et al. ${ }^{6}$ Three days before injection, guide screws (Plastics One) without stylets were implanted. After anesthesia induction and the incision, an 18-gauge needle was used to start drilling a hole 1.0 $\mathrm{mm}$ anterior to the coronal suture and $2.5 \mathrm{~mm}$ lateral to the sagittal suture. Another 18-gauge needle with half of the tip cut off was then used to enlarge the hole without puncturing the dura mater. The guide screw was inserted using the screwdriver provided (Plastics One) and fixed to the skull using dental cement (ESPE Adper Single Bond $2,3 \mathrm{M}$ ) that was thickened with bone cement (type 1 with gentamicin; Jorgensen Laboratories) immediately before application and cured with a 10-second application of 455$\mathrm{nm}$ light. The incision was closed with cyanoacrylate.

\section{U87-LucNeo Cell Injection}

For injections, U87-LucNeo cells were grown to $80 \%$ confluence, suspended with trypsin, counted, and resuspended in $5 \mu \mathrm{l}$ of Matrigel (BD Biosciences). The skin over the previously implanted guide screw was sterilized with ethanol. Cells were drawn up in a $10-\mu l$ Hamilton syringe with a 26-gauge large-bore needle and mounted on a stereotactic frame. The needle was used to puncture the skin and was inserted into the guide screw. The depth of insertion $(3.5 \mathrm{~mm})$ was controlled by an 18-gauge needle that was cut and placed over the syringe needle to provide a positive stop against the guide screw. Cells were injected over a period of 2 minutes, and then the needle was left in place for 1 minute before removal. The mice were screened with bioimaging 5 days and 14 days after injection to verify tumor establishment before beginning the siRNA injections.

\section{siRNA Treatment}

RNAi was initiated using our previously published siRNAs targeting HIF-1 $\alpha$ (siRNA-1589 and siRNA-1124), a negative control (siRNA-Neg), ${ }^{18}$ and another negative control that targets green fluorescent protein (siRNAGFP) (sense, 5'-UCCAGGAGCGCACCAUCdTdT; antisense, 5'-GAUGGUGCGCUCCUGGAdTdT). Over a 49-day period, the mice were given daily intratumoral injections of 25 pmol of siRNA complexed to EHCO, as described previously. ${ }^{60,61}$ The amount of siRNA to be injected was based on cell culture and previous tumor flank results. ${ }^{18}$ Each mouse was anesthetized with isoflurane ( $2 \%, 1 \mathrm{~L} /$ minute oxygen), and the scalp area was wiped with ethanol and RNase ZAP (Ambion). An RNase-free Hamilton syringe with a 26-gauge needle and a depthcontrol stop was used to inject $3 \mu \mathrm{l}$ of siRNA over 1 minute, directly through the skin and guide screw, into the implanted tumor. The injection experiments were repeated 3 times using groups of 10 mice for each siRNA.

Alternatively, mice were given 416.7 pmol of siRNA complexed to EHCO through continuously dispensing miniosmotic pumps (Alzet 1002; Durect) that dispense the siRNA-carrier complex at a rate of $0.25 \mu \mathrm{l} /$ hour over a period of 14 days. The pumps were made to dispense at the same depth and amount per week as the daily injections. The pumps were prepared and implanted as directed by the manufacturer. The catheter tubing included in the braininfusion kit was cut to a length of approximately $27 \mathrm{~mm}$ to be flexible enough for normal mouse activity. Special brain cannulae (Plastics One) were made of non-photon-emitting plastic so as not to interfere with bioluminescent image acquisition. A bur hole for each cannula was made with a 26-gauge needle, and the cannula was fixed to the skull with dental cement as in the guide-screw protocol above.

\section{In Vivo Imaging}

Mice were injected with a sterile solution of D-luciferin (6 mg/kg; Gold Biotechnology), ketamine $(8 \mathrm{mg} / \mathrm{kg})$, and xylazine $(0.8 \mathrm{mg} / \mathrm{kg})$ (both from Sigma-Aldrich) in 0.25 $\mathrm{ml}$ of $1 \times$ phosphate-buffered saline $(\mathrm{pH}$ 7.4). The mice were imaged with a Xenogen IVIS 100 using medium binning and a field of view of $10 \mathrm{~cm}$, with a 10- to 30 -second exposure exactly 10 minutes after injection. After acquisition, the images were analyzed using the LivingImage software provided with the IVIS 100 . The average radiance and total flux for the individual mice were used for data analysis.

\section{Fluorescently Labeled siRNA Injections}

Cy3.5 fluorescently labeled siRNA oligonucleotides from the University of Utah Synthesis Core were injected using the same siRNA-injection protocol mentioned previously, except the solution also contained Hoechst 33342 $(10 \mu \mathrm{g} / \mathrm{ml})$ to label nuclei and confirm the injection location on tissue sections. Mouse brains were harvested 1 and 5 minutes after injection, fixed in buffered formalin overnight, paraffin mounted, and stained with $\mathrm{H} \& \mathrm{E}$. Background autofluorescence was quenched by soaking in a solution of $10 \mathrm{mM} \mathrm{CuSO}_{4}$ dissolved in $50 \mathrm{mM} \mathrm{NH}_{4} \mathrm{Ac}$ (pH 5) for 1 hour. $^{48}$ 
Immunohistochemistry

HIF-1 $\alpha$, VEGF, CA-IX, c-MET, and GLUT-1

For HIF-1 $\alpha$ IHC, the Catalyzed Signal Amplification System (Dako), based on streptavidin-biotin-horseradish peroxidase complex formation, was used according to the manufacturer's recommended protocol. The primary antibody used was H1 $\alpha 67$ (Novus Biologicals) at a dilution of 1:1000. Nuclei were lightly counterstained with toluidine blue.

Heat-induced epitope retrieval was performed for cMET and GLUT-1 in a citrate buffer (Vector Laboratories) by heating for 7 minutes in a microwave pressure cooker on high and then cooling for 20 minutes before opening. For VEGF, CA-IX, c-MET, and GLUT-1, specimens were incubated with anti-VEGF Ab-1 polyclonal antibody (1:50 dilution; Calbiochem), anti-CA-IX goat polyclonal antibody (1:200 dilution; Santa Cruz Biotechnology), anti-cMET rabbit polyclonal antibody (1:100 dilution; Abcam), or rabbit anti-GLUT-1 (1:100 dilution; Santa Cruz Biotechnology). Secondary antibodies and avidin-biotin complex incubations were performed using a Vectastain $\mathrm{ABC}$ kit (Vector Laboratories). The final reaction was treatment of the sections in a peroxidase substrate solution, 3,3'-diaminobenzidine tetrahydrochloride (DAB; Vector Laboratories). Counterstaining was performed with toluidine blue. All slides were examined under $\times 200$ magnification with an Olympus BX41 microscope and were scored by 2 investigators blinded to mouse treatment information and tumor grade. The IHC analyses of HIF$1 \alpha$, VEGF, CA-IX, c-MET, and GLUT-1 were scored from 0 to $4(0,0 \%$ to $<25 \%$ staining; $1,25 \%$ to $<50 \% ; 2,50 \%$ to $<75 \% ; 3,75 \%$ to $<100 \%$; and $4,100 \%$ ) on the basis of the number of cells stained in a given field and averaged. ${ }^{14}$

\section{MIB-1 (Proliferation Index)}

To determine the proliferation index (PI), the primary antibody Ki-67 (clone MIB-1, dilution of 1:160) was used with a secondary antibody (dilution of 1:300; mouse fragment antigen-binding [Fab]; Dako). Detection was performed using the IView DAB detection kit, and counterstaining was performed with hematoxylin (Ventana ES, Ventana Medical Systems). The PI was calculated by taking 6 random pictures representative of each slide at $\times 400$ (10 ocular $\times 40$ objective) magnification with an Olympus MicroFire camera. Images were transferred to Image-Pro Plus 5.0, and a low-pass large spectral filter was applied. By using the count/size measurement feature, the MIB-1stained cells (brown) were counted with the manual intensity range-selection tool by histogram-based segmentation. The background-stained cells (blue) were counted in the same manner. The PI was calculated as the number of MIB-1-stained cells divided by the total number of cells in the field [(number of brown-stained cells)/(number of brown-stained cells + number of blue-stained cells)].

\section{Microvascular Density Index}

The slides for the microvascular density (MVD) index analysis were prepared using the same steps as described above for the MIB-1 analysis, except that they were pretreated with Factor VIII (rabbit polyclonal) protease 2
(Ventana Medical Systems) and the primary antibody (dilution of 1:100). The slides were then soaked in a secondary antibody (dilution of 1:300; mouse Fab). The MVD index was calculated on the basis of a previously published method ${ }^{14}$ using an Olympus MicroFire camera by 2 blinded investigators.

\section{Statistical Analysis}

Prism GraphPad 6 was used to analyze the in vivo luciferase and IHC data by one-way ANOVA with Tukey's multiple-comparisons test. The standard statistical significance level $(\mathrm{p}<0.05)$ was used. Kaplan-Meier survival data were analyzed by the log-rank (Mantel-Cox) and Gehan-Breslow-Wilcoxon tests.

\section{Results}

EHCO is a pH-sensitive amphiphilic lipid that is able to protonate in the acidic environment of endocytic compartments. This protonation promotes membrane bilayer disruption and siRNA release into the cytoplasm, where much of the cellular RNAi machinery is located. The confocal images presented in Fig. 1 verified the occurrence of this process in the U87-LucNeo cells used in this study. Forty-eight hours after transfection, it is evident that the siRNA (labeled with Alexa Fluor 488; Fig. 1A) escaped from the lysosomal compartments (labeled with LysoTracker Red; Fig. 1B) and dispersed throughout the cell. Colocalization of the lysosomes and siRNA suggests that the EHCO-siRNA nanoparticles were taken up through endocytosis and shuttled through endosomal and lysosomal compartments (Fig. 1C).

The ability of EHCO-siRNA nanoparticles to diffuse into the tumor tissue after injection was assessed via fluorescent histology. Figure 2 shows formalin-fixed brain sections that display effective delivery to the tumor site 1 minute after the local injection of Cy3.5-labeled EHCOsiRNA (Fig. 2A). Codelivery of Hoechst 33342 highlights the extent of diffusion at the injection site. As shown in Fig. 2B, the EHCO-siRNA nanoparticles began to permeate the local tissue after 5 minutes, suggesting that the proposed therapy would effectively deliver anti-HIF-1 $\alpha$ siRNA into solid tumors.

Once the functionality and delivery of the EHCOsiRNA nanoparticles were established, daily intratumoral injections of EHCO-siRNA were administered over a 49-day period. Tumor growth was monitored with bioluminescent imaging using U87-LucNeo cells containing a constitutive luciferase reporter construct. This method was previously found to be an accurate approach for estimating tumor growth in this brain tumor model system. ${ }^{57}$ Tumor growth was monitored for 2 weeks before beginning treatment. Figure 3 shows representative examples of these imaging ( $\mathrm{radiance}\left[\mathrm{p} / \mathrm{s} / \mathrm{cm}^{2} / \mathrm{sr}\right]$ ) data for U87-LucNeo glioma-bearing mice that were injected with a control siRNA (siRNA-Neg or siRNA-GFP) or one that knocks down HIF-1 $\alpha$ (siRNA-1589 or siRNA-1124) on Day 1 of imaging (14 days after implantation) or on Day 49. Mice that were injected with siRNAs targeting HIF-1 $\alpha$ showed significantly less tumor growth than those injected with scrambled or nontargeting siRNAs (Fig. 4). Tumor growth 

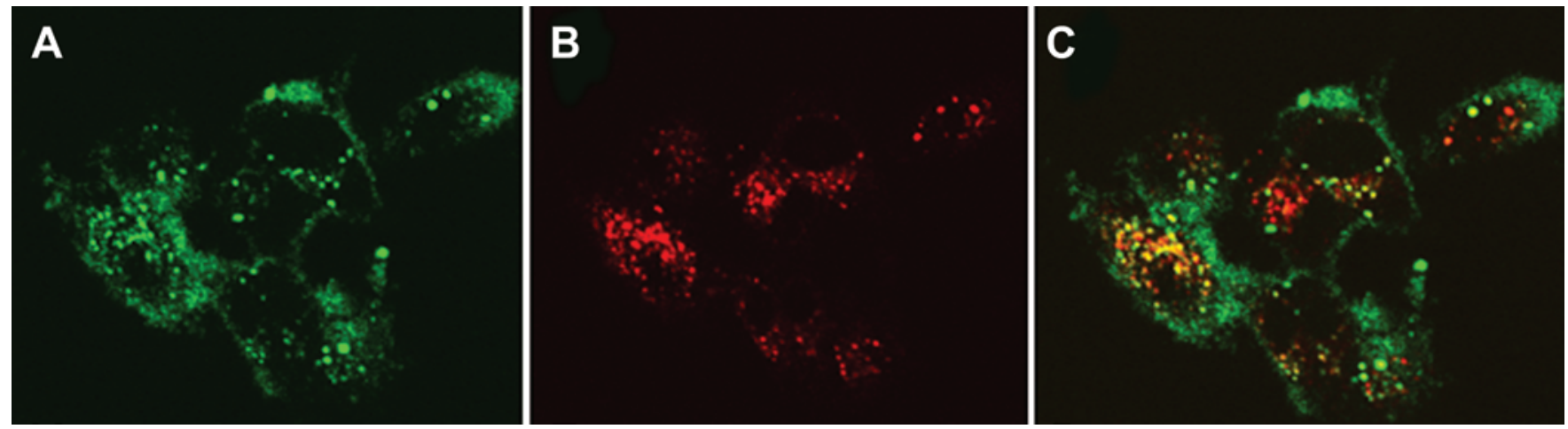

FIG. 1. Images of U87-LucNeo cells 48 hours after transfection using Alexa Fluor 488-labeled siRNA (A) or LysoTracker Red DND-99 (B) and these two images merged (C). Original magnification $\times 1000$.

was reduced by $79 \%$ as measured by luciferase activity ( $\mathrm{p}$ $=0.0027$, one-way ANOVA) when compared with the negative controls (siRNA-Neg and siRNA-GFP). There were no significant differences between these 2 control groups or between the 2 treatment groups ( $p>0.05$ ).

A Kaplan-Meier survival analysis showed that survival was also increased significantly in the HIF-1 $\alpha$ knockdown siRNA-1124 group compared with the GFP knockdowninjection group (Fig. 5 left), as well as in the PEG-EHCO groups treated via osmotic pumps (Fig. 5 right). It is interesting to note that when siRNA was administered by osmotic pumps to mimic CED, the non-PEGylated siRNA-1589 still had some effect beyond the GFP control, although it was not significantly different. However, PEGylation of the EHCO-1589 nanoparticles resulted in significantly longer survival of tumor-bearing mice in comparison with the PEGylated GFP control. This result suggests that the addition of PEG to EHCO enhances siRNA stability and improves its effectiveness in vivo. We have found that PEG-EHCO-siRNA complexes are still functional for up to 2 weeks when stored in cell culture medium with $10 \%$ bovine serum at $37^{\circ} \mathrm{C}$ (data not shown). Although no direct comparison can be made because of different experimental conditions, a comparison of the time points at which the different treatment groups began to separate in Fig. 4 (3040 days) and Fig. 5 right (10-20 days) suggests that the continuous PEG-EHCO-siRNA treatment was more effective. Special consideration must be given to the fact that the survival data were also influenced by the morbidity involved with the pump-implanting procedure. Approximately $15 \%$ of the mice experienced infections or problems with the cannula becoming obstructed by scar tissue. These data correlate with what we found previously with other siRNAEHCO complex-protecting moieties. ${ }^{61}$

IHC analysis revealed that the levels of several transcriptional targets of HIF-1 $\alpha$ were reduced significantly in the tumors of mice treated with siRNA knocking down HIF-1 $\alpha$. Figure 6 shows representative IHC evidence that HIF-1, GLUT-1, CA-IX, c-MET, and VEGF were all reduced significantly in tumors treated with HIF-1 $\alpha$-target-
A

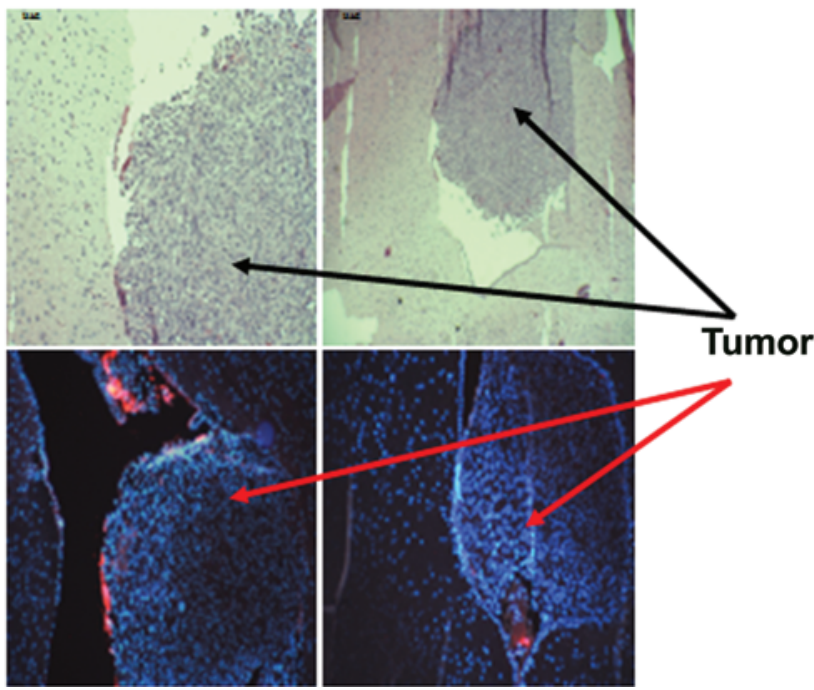

B

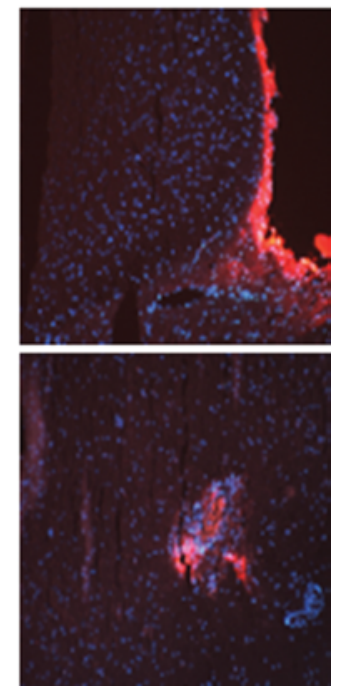

FIG. 2. EHCO-siRNA labeled with Cy3.5 (red) was injected as described in a solution of Hoechst $33342(10 \mu \mathrm{g} / \mathrm{ml})$ to label the nuclei (blue). Brain tissue was harvested at 1 minute $(A)$ and 5 minutes $(B)$ after injection. Note the increased diffusion of siRNA into the tissue at 5 minutes. Original magnification $\times 200(A)$ and $\times 400(B)$. 
EHCO-1124, day 1
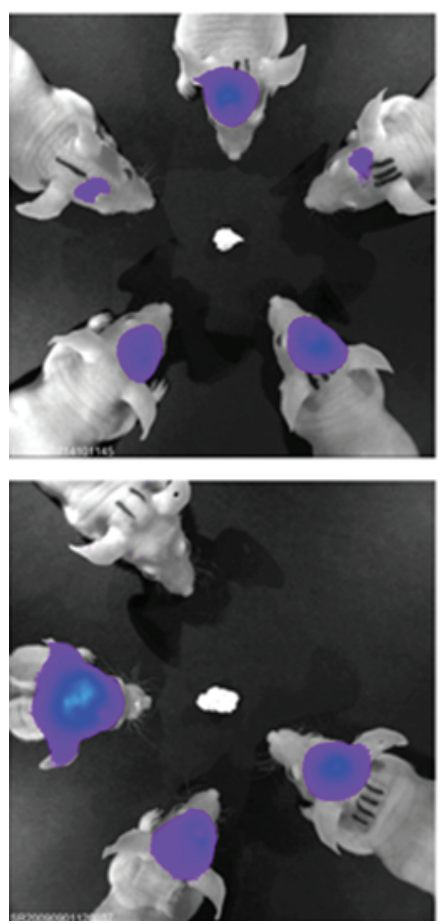

EHCO-Neg, day 1
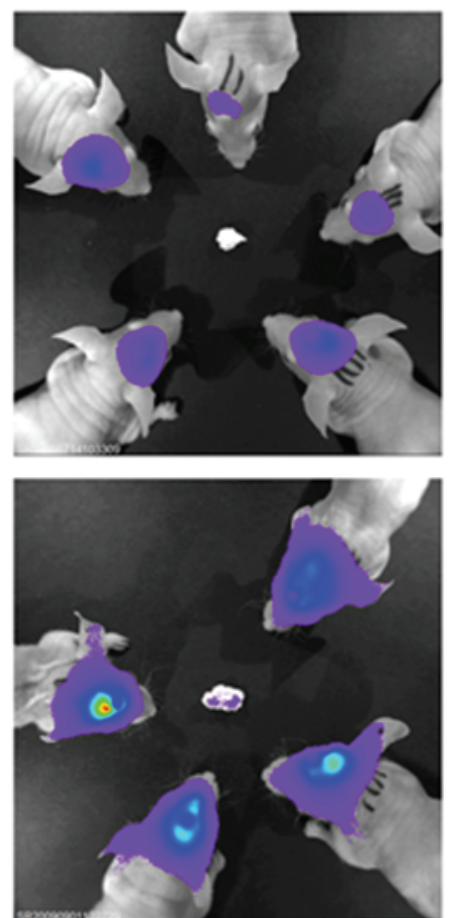

EHCO-GFP, day 1
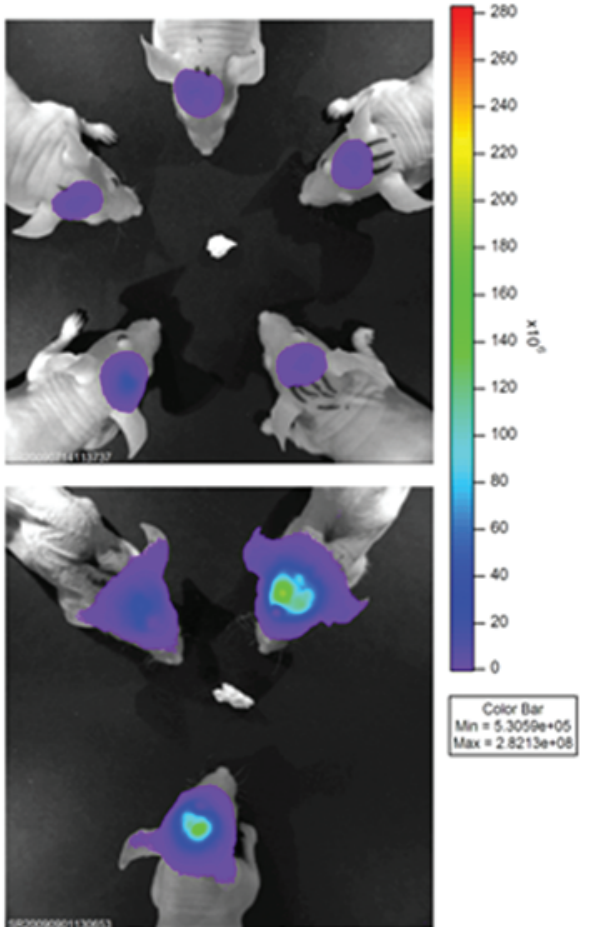

FIG. 3. Comparison of luciferase activity on Day 1 (upper images) with that on Day 49 (lower images) in tumors treated with antiHIF-1 siRNA-1124, or the control siRNA-GFP or siRNA-Neg. The image color scale is in radiance $\left(\mathrm{p} / \mathrm{s} / \mathrm{cm}^{2} / \mathrm{sr}\right)$. Figure is available in color online only.

ing siRNAs compared with the controls. Proliferation and angiogenesis were also reduced significantly, as shown by the reduction of MIB-1 staining and MVD (Fig. 7). Representative images of IHC tissue slides are shown for comparison. The reduction of all of these HIF-1 target levels in our treatment groups is strong evidence that our siRNA-delivery method is able to reduce HIF-1 $\alpha$ protein levels sufficiently to affect tumor growth in vivo.

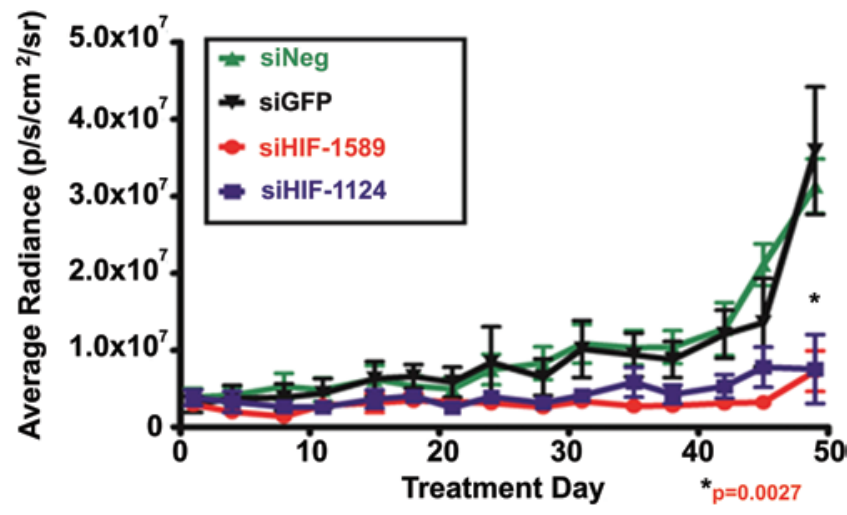

FIG. 4. The normalized average radiance graph showing normal tumor growth in mice injected intracranially (daily injections) with the negative control siRNA-Neg or siRNA-GFP. siRNA-1124 and siRNA-1589 show a significant decrease in radiance compared with the controls. Figure is available in color online only.

\section{Discussion}

To our knowledge, this is the first report to describe the use of an siRNA to knock down HIF-1 $\alpha$ in an orthotopic xenograft mouse model. Previously, we and others $\mathrm{F}^{18,61}$ showed that RNAi can effectively knock down HIF-1 $\alpha$ and affect tumor growth in a mouse flank tumor model; however, there are some concerns about the accuracy of that model, given the fundamental differences between the brain and flank environments. ${ }^{3}$ Other studies have used short hairpin RNA-expressing cells to knock down HIF$1 \alpha$ constitutively before implantation into animals. ${ }^{39}$ This approach is not a clinically viable option for treating patients, and it does not reflect how their tumors may respond when targeting HIF-1 $\alpha$ after the tumor has developed in an environment in which HIF-1 $\alpha$ is freely expressed. Here, we have demonstrated the ability to reduce tumor growth through the reduction of HIF-1 $\alpha$ via an injected transient EHCO-siRNA complex that is well tolerated by immunocompromised mice in vivo.

Intracellular release of siRNA from nanoparticles is essential for the success of any therapeutic RNAi protocol. After uptake into the cell via endocytosis, the delivery system must have the ability to facilitate siRNA escape from the endosomal-lysosomal pathway before degradation occurs. The data presented here indicate that EHCO does this effectively in a xenograft brain tumor mouse model, correlating with our previous in vivo and in vitro studies. ${ }^{60,61}$ Results of those studies showed that PEGylation of the siRNA-EHCO nanoparticles significantly reduced non- 

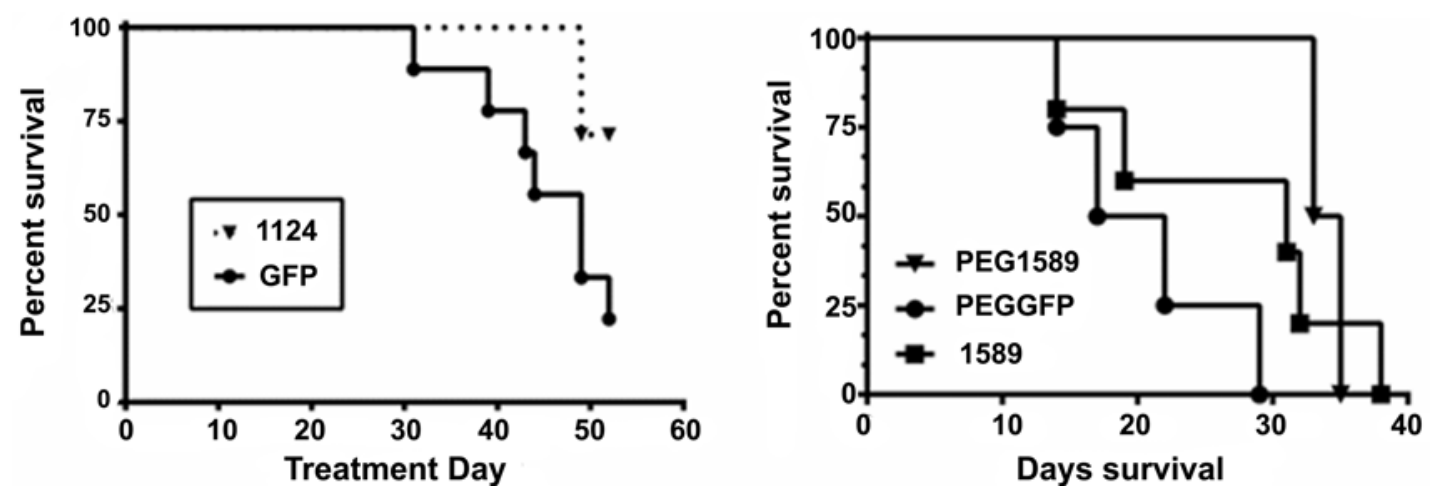

FIG. 5. Left: Survival curve of mice injected intracranially on a daily schedule. The siRNA-1124-injected group survived significantly longer $(p=0.04)$. Right: Mice treated with Alzet osmotic pumps dispensing $0.25 \mu$ l/hour to mimic CED. Groups were treated with EHCO-siRNA and PEGylated EHCO-siRNA. Mice in the PEG-EHCO-1589 group lived significantly longer than those in the PEG-GFP group $(p=0.0114)$. The pumps were removed on Day 14.

specific cell uptake. Here, we also provide evidence that PEGylation increases the in vivo effectiveness of EHCOsiRNA-mediated RNAi. Others have shown that PEG provides an aqueous shield around the nanoparticle, which decreases opsonization and macrophage recognition, resulting in a longer nanoparticle blood residence time. ${ }^{19}$ The ability of EHCO-siRNA complexes to diffuse into tumors after localized injections and the increased effectiveness of PEG-EHCO-siRNA in an animal model mimicking CED promise a delivery modality for treating solid tumors that is clinically relevant. Since its development in 1994, CED has become a popular investigational modality for treating brain tumors. By using positive pressure and continuous metered flow, delivery is enhanced over passive diffusion. Several clinical trials of CED have had mixed results, ${ }^{2}$ which emphasizes the need for additional development in this approach, such as developing more effective delivery agents such as PEG-EHCO. Because there is evidence that repeated exposure to PEG can invoke an immune response, which results in rapid clearance from the blood on repeated exposures,, 23 additional work in an immunocompetent model is necessary so that the full range of immune responses to PEG-EHCO-siRNA complexes can be determined.

The results we present here confirm that perturbing the HIF-1 $\alpha$ cascade is a viable therapeutic target for treating glioblastoma. Indeed, many studies have already confirmed this finding when targeting individual parts of the glycolytic pathway, such as pyruvate dehydrogenase kinase (PDK), ${ }^{56}$ isocitrate dehydrogenase 2 (IDH2), ${ }^{63}$ lactate dehydrogenase A (LDHA), ${ }^{33}$ and hexokinase II (HKII). ${ }^{64}$ A recently completed Phase I clinical trial that used HIF$1 \alpha$ inhibitor PX-478 for the treatment of advanced solid tumors showed promising results. ${ }^{34}$ Another trial to test the efficacy of DCA as an indirect HIF-1 inhibitor is currently underway. ${ }^{49}$ It is likely that the lack of tumor growth we report here is a result of the decrease in the level of one or more of the many HIF-1 $\alpha$ targets and not just a reduction of the HIF-1 $\alpha$ level itself. Each of these HIF$1 \alpha-$ controlled genes will need to be evaluated by specific targeting to sort out its contribution to the overall reduction of growth.

One of the possible consequences of reducing VEGF through targeting HIF-1 $\alpha$, one of its main activators, is increased tumor invasion. ${ }^{25}$ Studies of glioblastoma treated with bevacizumab, a VEGF-A inhibitor, have revealed increased invasion and matrix metalloproteinase expression. ${ }^{16}$ It has been proposed that this is mediated by the receptor tyrosine kinase c-MET, which is also upregulated in response to bevacizumab therapy. ${ }^{38}$ When treated with c-MET inhibitors, various lung cancer cell types lose their invasive phenotypes, ${ }^{32}$ and broad-spectrum kinase inhibitors have been shown to inhibit bevacizumab-induced invasion in gliomas..$^{21}$ HIF-1 is a known activator of c-MET in many cell types, including gliomas, and is thought to play a role in their invasiveness. ${ }^{8,13}$ This reduced c-MET activity may explain the lack of invasion that we observed in the HIF-1 $\alpha$ knock-down mice (data not shown). Although the cell line that we used has been criticized for its reduced invasive properties in our intracranial model, it does express scatter factor/hepatocyte growth factor $(\mathrm{SF} /$ HGF) and c-MET. ${ }^{30}$ Others have used these U87 cells in a xenograft mouse model and found that specific c-MET antagonists cause dose-dependent tumor growth inhibition..$^{41}$ Our results show that targeting HIF-1 $\alpha$ leads to reduced c-MET protein levels, which corroborates the results of studies that have shown that this also occurs in breast and lung cancer cell lines. ${ }^{2}$ It may be that disrupting HIF-1 function in gliomas, and thus c-MET, will not lead to the same invasion and metastasis encountered in anti-VEGF therapy. This seems to be the case in lung cancer, in which inhibiting HIF-1 activity blocked both cancer growth and metastases in mice. ${ }^{51}$

When c-MET is inhibited in glioblastoma xenografts, the tumor loses the ability to be propagated serially, indicating that c-MET plays a role in tumor-propagating stem-like cells. ${ }^{43}$ There is evidence that many stem cells are localized in hypoxic areas, supporting the hypothesis that hypoxia might be important for the undifferentiated phenotype of stem/precursor cells..$^{20,35}$ This hypoxic niche within glioblastoma is a potential therapeutic target for these devastating tumors. We propose that by targeting HIF-1 $\alpha$, these tumor-initiating cells are limited in their ability to propagate, resulting in the significant reduction in in vivo tumor growth reported here. 
A
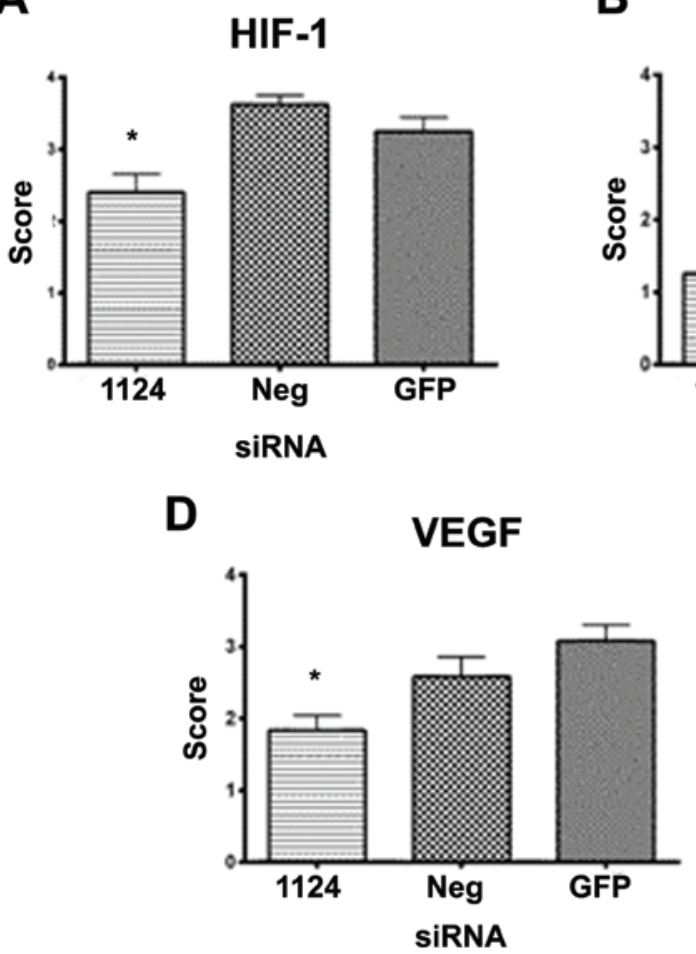

D

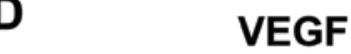

$\mathbf{F}$

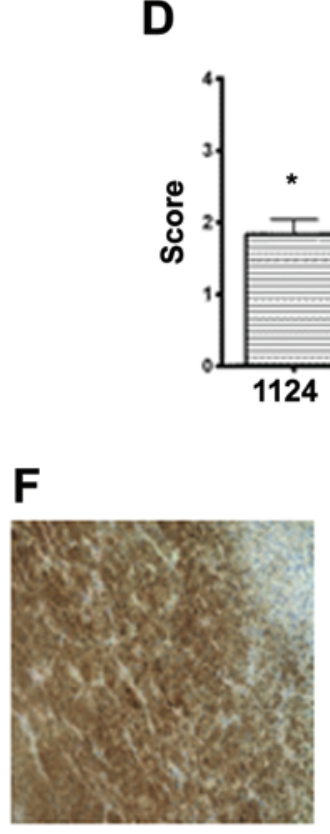

G

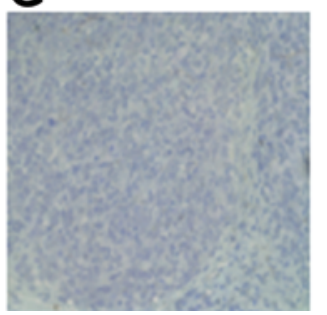

H

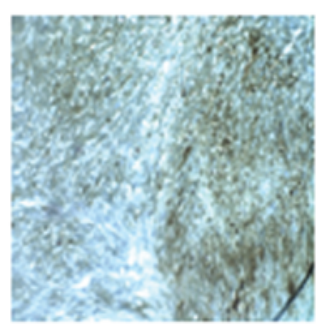

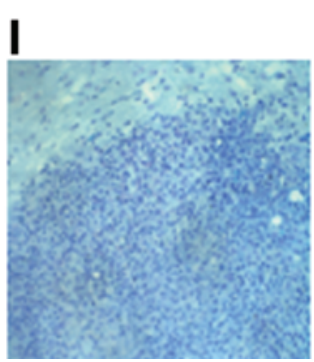

B

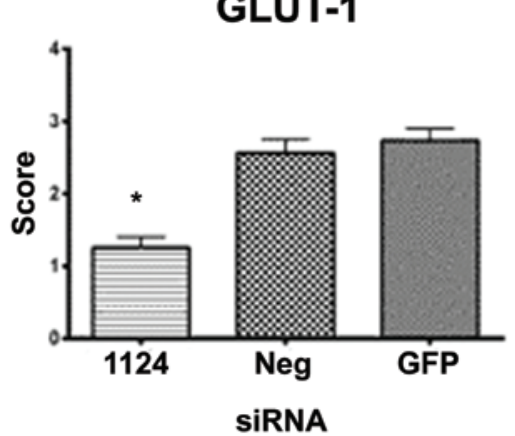

C

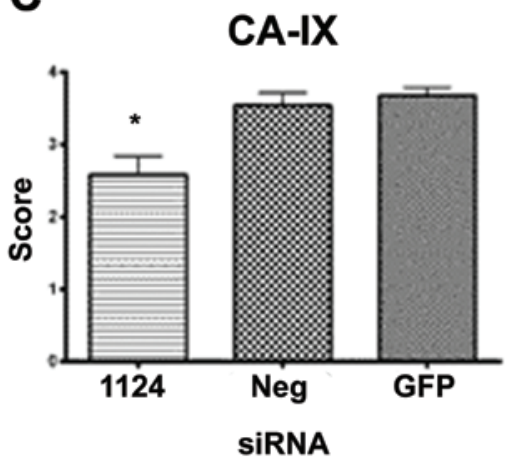

E
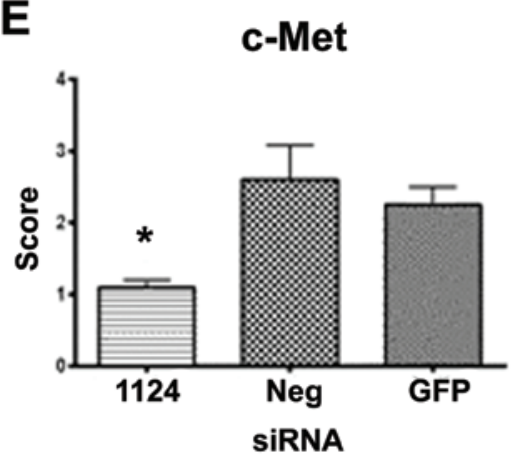

$\mathbf{L}$

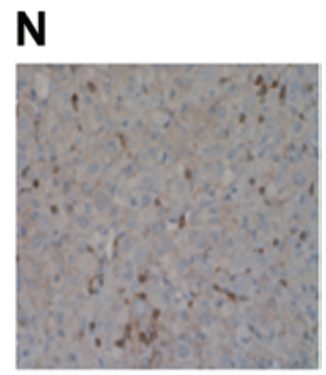

K

M

0

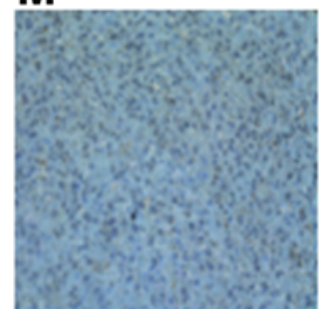

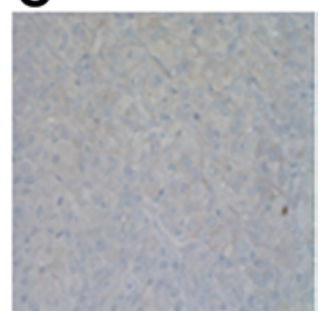

FIG. 6. Quantification of HIF-1 and some of its transcriptional targets after either HIF-1 $\alpha$-siRNA or control siRNA treatments in mouse intracranial U87 tumors analyzed by IHC (A-E). Representative positive (F) and negative (G) tissue stained (horseradish peroxidase-DAB stain) for HIF-1 ( $\left.{ }^{*} p=0.002\right)$. Positive (H) and negative (I) GLUT-1-stained tissue ( $\left.{ }^{*} p<0.001\right)$. Positive (J) and negative (K) CA-IX $\left({ }^{*} p=0.006\right)$. Positive $(\mathrm{L})$ and negative (M) VEGF $\left({ }^{*} p=0.008\right)$. Positive $(\mathrm{N})$ and negative (O) $c-M E T\left({ }^{*} p=\right.$ $0.0067)$. Brown staining indicates the presence of the specified protein. Magnification in all images, $\times 200$. Figure is available in color online only.

\section{Conclusions}

We have demonstrated a clinically relevant method for treating glioblastoma in an orthotopic mouse model by specifically targeting HIF-1 $\alpha$ with RNAi through the novel delivery agent EHCO. This method was also shown to have direct application through a model of CED using osmotic pumps, resulting in smaller tumors and increased survival. Evidence that c-MET was also reduced indicates that targeting HIF-1 $\alpha$ may be an effective means of reducing glioblastoma invasiveness. Additional investigation in an immunocompetent model is needed to assess the immune system response to EHCO-siRNA complexes.

\section{Acknowledgments}

We thank Kristin Kraus, MSc, for the editing and insight provided, the summer research and intern programs at the University 
A

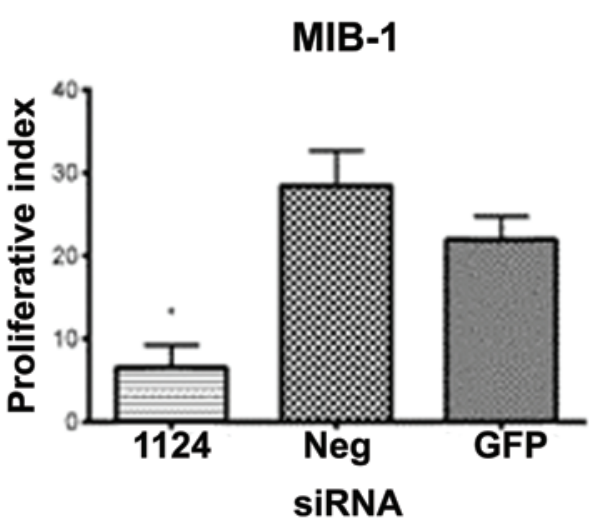

D

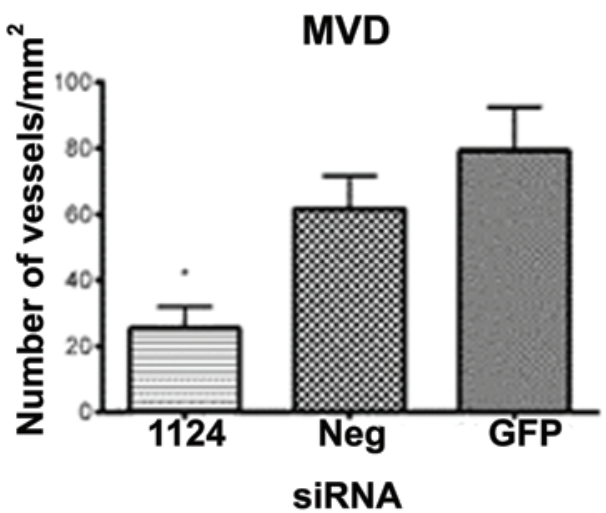

B

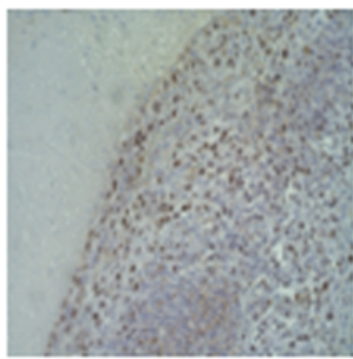

$\mathbf{E}$

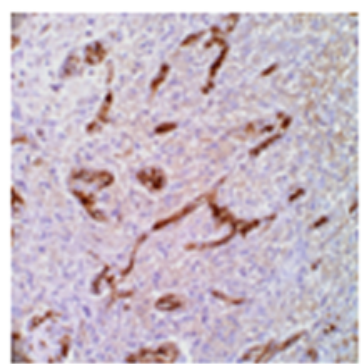

C

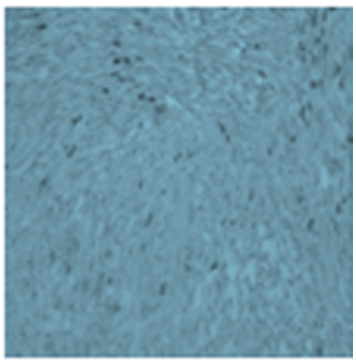

$\mathbf{F}$

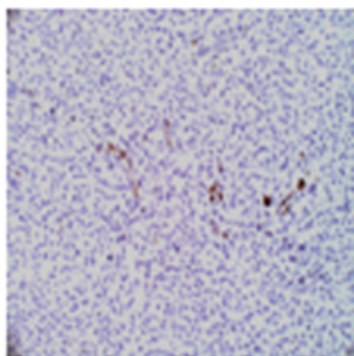

FIG. 7. A: Quantification of MIB-1 index after either HIF-1 $\alpha$-siRNA or control siRNA treatments in mouse intracranial U87 tumors analyzed by $\mathrm{IHC}\left({ }^{*}, p<0.0001\right)$. B and C: Representative positive $(B)$ and negative $(C)$ tumors stained (horseradish peroxidaseDAB stain) for MIB-1. D: Quantification of tumor MVD $\left({ }^{*}, p=0.001\right)$. E and F: Positive (E) and negative (F) MVD (factor VIII) staining. Brown staining indicates the presence of the specified protein. Original magnification $\times 200$. Figure is available in color online only.

of Utah School of Medicine and Huntsman Cancer Institute, which provided many of the hands to do the experiments, and the University of Utah DNA/peptide synthesis core facility for producing the siRNA used in these experiments.

\section{References}

1. Behlke MA: Progress towards in vivo use of siRNAs. Mol Ther 13:644-670, 2006

2. Bidros DS, Liu JK, Vogelbaum MA: Future of convectionenhanced delivery in the treatment of brain tumors. Future Oncol 6:117-125, 2010

3. Blouw B, Song H, Tihan T, Bosze J, Ferrara N, Gerber HP, et al: The hypoxic response of tumors is dependent on their microenvironment. Cancer Cell 4:133-146, 2003

4. Blow N: Small RNAs: delivering the future. Nature 450:1117-1120, 2007

5. Brat DJ, Mapstone TB: Malignant glioma physiology: cellular response to hypoxia and its role in tumor progression. Ann Intern Med 138:659-668, 2003

6. Brockmann MA, Westphal M, Lamszus K: Improved method for the intracerebral engraftment of tumour cells and intratumoural treatment using a guide screw system in mice. Acta Neurochir (Wien) 145:777-781, 2003

7. Chi JT, Wang Z, Nuyten DS, Rodriguez EH, Schaner ME, Salim A, et al: Gene expression programs in response to hypoxia: cell type specificity and prognostic significance in human cancers. PLoS Med 3:e47, 2006
8. Comito G, Calvani M, Giannoni E, Bianchini F, Calorini L, Torre E, et al: HIF-1 $\alpha$ stabilization by mitochondrial ROS promotes Met-dependent invasive growth and vasculogenic mimicry in melanoma cells. Free Radic Biol Med 51:893904, 2011

9. Dams ET, Laverman P, Oyen WJ, Storm G, Scherphof GL, van Der Meer JW, et al: Accelerated blood clearance and altered biodistribution of repeated injections of sterically stabilized liposomes. J Pharmacol Exp Ther 292:1071-1079, 2000

10. de Fougerolles A, Vornlocher HP, Maraganore J, Lieberman $\mathrm{J}$ : Interfering with disease: a progress report on siRNA-based therapeutics. Nat Rev Drug Discov 6:443-453, 2007

11. Dolecek TA, Propp JM, Stroup NE, Kruchko C: CBTRUS statistical report: primary brain and central nervous system tumors diagnosed in the United States in 2005-2009. Neuro Oncol 14 Suppl 5:v1-v49, 2012 (Erratum in Neuro Oncol 15:646-647, 2013)

12. Dubey R, Levin MD, Szabo LZ, Laszlo CF, Kushal S, Singh $\mathrm{JB}$, et al: Suppression of tumor growth by designed dimeric epidithiodiketopiperazine targeting hypoxia-inducible transcription factor complex. J Am Chem Soc 135:4537-4549, 2013

13. Eckerich C, Zapf S, Fillbrandt R, Loges S, Westphal M, Lamszus K: Hypoxia can induce c-Met expression in glioma cells and enhance $\mathrm{SF} / \mathrm{HGF}$-induced cell migration. Int J Cancer 121:276-283, 2007

14. Flynn JR, Wang L, Gillespie DL, Stoddard GJ, Reid JK, 
Owens J, et al: Hypoxia-regulated protein expression, patient characteristics, and preoperative imaging as predictors of survival in adults with glioblastoma multiforme. Cancer 113:1032-1042, 2008

15. Forsythe JA, Jiang BH, Iyer NV, Agani F, Leung SW, Koos $\mathrm{RD}$, et al: Activation of vascular endothelial growth factor gene transcription by hypoxia-inducible factor 1 . Mol Cell Biol 16:4604-4613, 1996

16. Furuta T, Nakada M, Misaki K, Sato Y, Hayashi Y, Nakanuma Y, et al: Molecular analysis of a recurrent glioblastoma treated with bevacizumab. Brain Tumor Pathol 31:32-39, 2014

17. Gadducci A, Guerrieri ME, Greco C: Tissue biomarkers as prognostic variables of cervical cancer. Crit Rev Oncol Hematol 86:104-129, 2013

18. Gillespie DL, Whang K, Ragel BT, Flynn JR, Kelly DA, Jensen RL: Silencing of hypoxia inducible factor-1alpha by RNA interference attenuates human glioma cell growth in vivo. Clin Cancer Res 13:2441-2448, 2007

19. Gomes-da-Silva LC, Fonseca NA, Moura V, Pedroso de Lima MC, Simões S, Moreira JN: Lipid-based nanoparticles for siRNA delivery in cancer therapy: paradigms and challenges. Acc Chem Res 45:1163-1171, 2012

20. Heddleston JM, Hitomi M, Venere M, Flavahan WA, Yang K, Kim Y, et al: Glioma stem cell maintenance: the role of the microenvironment. Curr Pharm Des 17:2386-2401, 2011

21. Huveldt D, Lewis-Tuffin LJ, Carlson BL, Schroeder MA, Rodriguez F, Giannini C, et al: Targeting Src family kinases inhibits bevacizumab-induced glioma cell invasion. PLoS ONE 8: 56505,2013

22. Ino Y, Yamazaki-Itoh R, Oguro S, Shimada K, Kosuge T, Zavada J, et al: Arginase II expressed in cancer-associated fibroblasts indicates tissue hypoxia and predicts poor outcome in patients with pancreatic cancer. PLoS ONE 8:e55146, 2013

23. Ishida T, Ichihara M, Wang X, Yamamoto K, Kimura J, Majima E, et al: Injection of PEGylated liposomes in rats elicits PEG-specific IgM, which is responsible for rapid elimination of a second dose of PEGylated liposomes. J Control Release 112:15-25, 2006

24. Jensen RL: Brain tumor hypoxia: tumorigenesis, angiogenesis, imaging, pseudoprogression, and as a therapeutic target. J Neurooncol 92:317-335, 2009

25. Keunen O, Johansson M, Oudin A, Sanzey M, Rahim SA, Fack F, et al: Anti-VEGF treatment reduces blood supply and increases tumor cell invasion in glioblastoma. Proc Natl Acad Sci U S A 108:3749-3754, 2011

26. Kietzmann T, Krones-Herzig A, Jungermann K: Signaling cross-talk between hypoxia and glucose via hypoxiainducible factor 1 and glucose response elements. Biochem Pharmacol 64:903-911, 2002

27. Kim SS, Garg H, Joshi A, Manjunath N: Strategies for targeted nonviral delivery of siRNAs in vivo. Trends Mol Med 15:491-500, 2009

28. Kleihues P, Louis DN, Scheithauer BW, Rorke LB, Reifenberger G, Burger PC, et al: The WHO classification of tumors of the nervous system. J Neuropathol Exp Neurol 61:215-229, 2002

29. Lal A, Peters H, St Croix B, Haroon ZA, Dewhirst MW, Strausberg RL, et al: Transcriptional response to hypoxia in human tumors. J Natl Cancer Inst 93:1337-1343, 2001

30. Lal B, Xia S, Abounader R, Laterra J: Targeting the c-Met pathway potentiates glioblastoma responses to gamma-radiation. Clin Cancer Res 11:4479-4486, 2005

31. Lal S, Lacroix M, Tofilon P, Fuller GN, Sawaya R, Lang FF: An implantable guide-screw system for brain tumor studies in small animals. J Neurosurg 92:326-333, 2000

32. Lawrence RE, Salgia R: MET molecular mechanisms and therapies in lung cancer. Cell Adhes Migr 4:146-152, 2010
33. Le A, Cooper CR, Gouw AM, Dinavahi R, Maitra A, Deck LM, et al: Inhibition of lactate dehydrogenase A induces oxidative stress and inhibits tumor progression. Proc Natl Acad Sci U S A 107:2037-2042, 2010

34. Lee K, Kim HM: A novel approach to cancer therapy using PX-478 as a HIF-1 $\alpha$ inhibitor. Arch Pharm Res 34:15831585,2011

35. Lin Q, Lee YJ, Yun Z: Differentiation arrest by hypoxia. J Biol Chem 281:30678-30683, 2006

36. Lu H, Forbes RA, Verma A: Hypoxia-inducible factor 1 activation by aerobic glycolysis implicates the Warburg effect in carcinogenesis. J Biol Chem 277:23111-23115, 2002

37. Mao Q, Zhang Y, Fu X, Xue J, Guo W, Meng M, et al: A tumor hypoxic niche protects human colon cancer stem cells from chemotherapy. J Cancer Res Clin Oncol 139:211-222, 2013

38. McCarty JH: Glioblastoma resistance to anti-VEGF therapy: has the challenge been MET? Clin Cancer Res 19:16311633,2013

39. Méndez O, Zavadil J, Esencay M, Lukyanov Y, Santovasi D, Wang SC, et al: Knock down of HIF-1 $\alpha$ in glioma cells reduces migration in vitro and invasion in vivo and impairs their ability to form tumor spheres. Mol Cancer 9:133, 2010

40. Niclou SP, Fack F, Rajcevic U: Glioma proteomics: status and perspectives. J Proteomics 73:1823-1838, 2010

41. Olwill SA, Joffroy C, Gille H, Vigna E, Matschiner G, Allersdorfer A, et al: A highly potent and specific MET therapeutic protein antagonist with both ligand-dependent and ligand-independent activity. Mol Cancer Ther 12:24592471,2013

42. Onnis B, Rapisarda A, Melillo G: Development of HIF-1 inhibitors for cancer therapy. J Cell Mol Med 13 (9A):2780 2786, 2009

43. Rath P, Lal B, Ajala O, Li Y, Xia S, Kim J, et al: In vivo cMet pathway inhibition depletes human glioma xenografts of tumor-propagating stem-like cells. Transl Oncol 6:104-111, 2013

44. Reardon DA, Herndon JE II, Peters K, Desjardins A, Coan A, Lou E, et al: Outcome after bevacizumab clinical trial therapy among recurrent grade III malignant glioma patients. J Neurooncol 107:213-221, 2012

45. Richard DE, Berra E, Pouysségur J: Angiogenesis: how a tumor adapts to hypoxia. Biochem Biophys Res Commun 266:718-722, 1999

46. Robey IF, Lien AD, Welsh SJ, Baggett BK, Gillies RJ: Hypoxia-inducible factor- $1 \alpha$ and the glycolytic phenotype in tumors. Neoplasia 7:324-330, 2005

47. Sathornsumetee S, Cao Y, Marcello JE, Herndon JE II, McLendon RE, Desjardins A, et al: Tumor angiogenic and hypoxic profiles predict radiographic response and survival in malignant astrocytoma patients treated with bevacizumab and irinotecan. J Clin Oncol 26:271-278, 2008

48. Schnell SA, Staines WA, Wessendorf MW: Reduction of lipofuscin-like autofluorescence in fluorescently labeled tissue. J Histochem Cytochem 47:719-730, 1999

49. Semenza GL: HIF-1 mediates metabolic responses to intratumoral hypoxia and oncogenic mutations. J Clin Invest 123:3664-3671, 2013

50. Semenza GL: Intratumoral hypoxia, radiation resistance, and HIF-1. Cancer Cell 5:405-406, 2004

51. Semenza GL: Molecular mechanisms mediating metastasis of hypoxic breast cancer cells. Trends Mol Med 18:534543, 2012

52. Semenza GL: Regulation of mammalian $\mathrm{O} 2$ homeostasis by hypoxia-inducible factor 1. Annu Rev Cell Dev Biol 15:551578,1999

53. Solaini G, Sgarbi G, Baracca A: Oxidative phosphorylation in cancer cells. Biochim Biophys Acta 1807:534-542, 2011

54. Søndergaard KL, Hilton DA, Penney M, Ollerenshaw M, 
Demaine AG: Expression of hypoxia-inducible factor $1 \alpha$ in tumours of patients with glioblastoma. Neuropathol Appl Neurobiol 28:210-217, 2002

55. Sutendra G, Dromparis P, Kinnaird A, Stenson TH, Haromy A, Parker JM, et al: Mitochondrial activation by inhibition of PDKII suppresses HIF1a signaling and angiogenesis in cancer. Oncogene 32:1638-1650, 2013

56. Sutendra G, Michelakis ED: Pyruvate dehydrogenase kinase as a novel therapeutic target in oncology. Front Oncol 3:38, 2013

57. Szentirmai O, Baker CH, Lin N, Szucs S, Takahashi M, Kiryu S, et al: Noninvasive bioluminescence imaging of luciferase expressing intracranial U87 xenografts: correlation with magnetic resonance imaging determined tumor volume and longitudinal use in assessing tumor growth and antiangiogenic treatment effect. Neurosurgery 58:365-372, 2006

58. Vredenburgh JJ, Desjardins A, Reardon DA, Peters KB, Herndon JE II, Marcello J, et al: The addition of bevacizumab to standard radiation therapy and temozolomide followed by bevacizumab, temozolomide, and irinotecan for newly diagnosed glioblastoma. Clin Cancer Res 17:4119-4124, 2011

59. Wang GL, Jiang BH, Rue EA, Semenza GL: Hypoxiainducible factor 1 is a basic-helix-loop-helix-PAS heterodimer regulated by cellular O2 tension. Proc Natl Acad Sci U S A 92:5510-5514, 1995

60. Wang XL, Ramusovic S, Nguyen T, Lu ZR: Novel polymerizable surfactants with $\mathrm{pH}$-sensitive amphiphilicity and cell membrane disruption for efficient siRNA delivery. Bioconjug Chem 18:2169-2177, 2007

61. Wang XL, Xu R, Wu X, Gillespie D, Jensen R, Lu ZR: Targeted systemic delivery of a therapeutic siRNA with a multifunctional carrier controls tumor proliferation in mice. Mol Pharm 6:738-746, 2009

62. Warburg O: On the origin of cancer cells. Science 123:309314,1956
63. Wise DR, Ward PS, Shay JE, Cross JR, Gruber JJ, Sachdeva $\mathrm{UM}$, et al: Hypoxia promotes isocitrate dehydrogenasedependent carboxylation of $\alpha$-ketoglutarate to citrate to support cell growth and viability. Proc Natl Acad Sci U S A 108:19611-19616, 2011

64. Wolf A, Agnihotri S, Micallef J, Mukherjee J, Sabha N, Cairns R, et al: Hexokinase 2 is a key mediator of aerobic glycolysis and promotes tumor growth in human glioblastoma multiforme. J Exp Med 208:313-326, 2011

65. Xu RH, Pelicano H, Zhou Y, Carew JS, Feng L, Bhalla KN, et al: Inhibition of glycolysis in cancer cells: a novel strategy to overcome drug resistance associated with mitochondrial respiratory defect and hypoxia. Cancer Res 65:613-621, 2005

66. Zagzag D, Zhong H, Scalzitti JM, Laughner E, Simons JW, Semenza GL: Expression of hypoxia-inducible factor 1alpha in brain tumors: association with angiogenesis, invasion, and progression. Cancer 88:2606-2618, 2000

\section{Author Contributions}

Conception and design: Jensen, Gillespie. Acquisition of data: all authors. Analysis and interpretation of data: all authors. Drafting the article: Gillespie. Critically revising the article: all authors. Reviewed submitted version of manuscript: all authors. Approved the final version of the manuscript on behalf of all authors:

Jensen. Statistical analysis: Gillespie. Study supervision: Jensen.

\section{Correspondence}

Randy L. Jensen, Department of Neurosurgery, University of Utah, 175 N. Medical Dr. E, Salt Lake City, UT 84132. email: randy.jensen@hsc.utah.edu. 